\title{
Medium-induced transverse momentum broadening via forward dijet correlations
}

\author{
Jiangyong Jia $\odot,{ }^{1,2}$ Shu-Yi Wei®, ${ }^{3,4}$ Bo-Wen Xiao® ${ }^{5}$ and Feng Yuan ${ }^{6}$ \\ ${ }^{1}$ Chemistry Department, Stony Brook University, Stony Brook, New York 11794, USA \\ ${ }^{2}$ Physics Department, Brookhaven National Laboratory, Upton, New York 11796, USA \\ ${ }^{3}$ CPHT, CNRS, Ecole Polytechnique, Institut Polytechnique de Paris, \\ Route de Saclay, 91128 Palaiseau, France \\ ${ }^{4}$ European Centre for Theoretical Studies in Nuclear Physics and Related Areas (ECT*) \\ and Fondazione Bruno Kessler, Strada delle Tabarelle 286, I-38123 Villazzano (TN), Italy \\ ${ }^{5}$ Key Laboratory of Quark and Lepton Physics (MOE) and Institute of Particle Physics, \\ Central China Normal University, Wuhan 430079, China \\ ${ }^{6}$ Nuclear Science Division, Lawrence Berkeley National Laboratory, Berkeley, California 94720, USA
}

(Received 23 November 2019; accepted 3 May 2020; published 12 May 2020)

\begin{abstract}
Dijet azimuthal angle correlation is arguably one of the most direct probes of the medium-induced broadening effects. The evidence for such broadening, however, is not yet clearly observed within the precision of current midrapidity measurements at RHIC and the LHC. We show that the dijet correlation in forward rapidity from the future LHC RUN3, aided by forward detector upgrades, can reveal this broadening thanks to the steeper jet spectra, suppressed vacuum radiations and lower underlying event background, with a sensitivity comparable to that of the future high-luminosity $\mathrm{Au}+\mathrm{Au}$ run at RHIC. Dijet correlation measurements at the two facilities together can provide powerful constraints on the temperature dependence of medium transport properties.
\end{abstract}

DOI: 10.1103/PhysRevD.101.094008

\section{INTRODUCTION}

There has been a lot of evidence that the quark gluon plasma (QGP) has been created in the heavy-ion collisions at RHIC and the LHC. It also suggests that the QGP behaves like a nearly inviscid fluid and is opaque to colored probes. The properties and short-range structures of the QGP can be inferred from the scattering patterns of energetic partons/jets as they traverse the medium [1]. Previous measurements have revealed the "jet quenching" phenomena [2,3]: a strong in-medium modification in the yield, shape and correlation patterns for these jets [4-6]. Theoretical efforts in describing these results have led to the extraction of an important parameter $\hat{q}$ quantifying the transverse momentum $\left(p_{\mathrm{T}}\right)$ broadening of single hard parton, which also controls jet energy loss and in-medium splitting processes [7-16].

Unlike the typical multiple scattering process in QED, the jet probe is itself an evolving multi-body system, splitting into a shower of partons as it loses virtuality.

Published by the American Physical Society under the terms of the Creative Commons Attribution 4.0 International license. Further distribution of this work must maintain attribution to the author(s) and the published article's title, journal citation, and DOI. Funded by SCOAP ${ }^{3}$.
During its propagation and evolution in the QGP, the jet not only loses energy and momentum, but also accumulates $p_{\mathrm{T}}$-broadening through medium-induced radiation and scattering. While measurements of leading parton energy loss provide constrains on the $\hat{q}$ within a given model [17], they are not very sensitive to the mechanisms and formalism in the calculations. On the other hand, jet $p_{\mathrm{T}^{-}}$ broadening arising from overall deflection and in-medium parton shower should be directly sensitive to any microscopic structure of the QGP. One promising observable for this purpose is the dihadron or dijet azimuthal angle $\Delta \phi$ correlations [18-24]. In $p p$ collisions, the dijet $\Delta \phi$ correlation can be described using the Sudakov resummation framework established in Refs. [25-28]. In A + A collisions, the $\Delta \phi$ correlation is expected to be further broadened by jet-medium interactions, and this broadening, if measured, can directly constrain the $\hat{q}$. Due to large vacuum Sudakov contributions, current measurements [29-33] are statistically and systematically limited for a clear observation.

Besides the complexity associated with the jet probes, the medium is rapidly expanding and its properties are highly dynamical. The jet-medium interactions are sensitive to the full evolution of both the jet and the medium from the initial to the final state, which complicates the determination of medium properties at given temperature. 
Since the mediums created at RHIC and the LHC have different temperature and different space-time evolution, a combined analysis of the same observables at RHIC and the LHC provide important lever arm to disentangle the temperature dependence from dynamical evolution $[34,35]$. Such exercise has been successfully carried out for the extraction of $\eta / s$ and other bulk properties based on Bayesian analysis of soft particle observables [36]. In the jet sector, a simultaneous comparison to the leading hadron suppression at RHIC and the LHC was shown to reduce the certainty of $\hat{q}$, and even suggests a possible nonmonotonic temperature dependence [17]. This RHICLHC complementarity was also demonstrated for several full jet observables with the sPHENIX detector in the future RHIC run [34].

Another lever arm for extracting the temperature dependence of QGP properties and structures is also provided by comparing jet measurements at midrapidity at RHIC with those at forward-rapidity at LHC. This is because the medium produced at forward-rapidity may have a temperature closer to the medium produced at midrapidity at lower $\sqrt{s_{N N}}$, but with a very different space-time dynamics. Furthermore, the jet spectra and their flavor composition at forward rapidity may also resemble those at midrapidity at lower $\sqrt{s_{N N}}$. Therefore, the forward-rapidity measurements provide a different setup for disentangling the temperature dependence from dynamical evolution. Another favorable factor is that the underlying event background (UE) fluctuations decreases at forwardrapidity, and the $d E_{\mathrm{T}} / d \eta$ at $|\eta| \sim 4$ is about $\times 2$ smaller than $\eta \sim 0$ at the LHC [37]. The measurements of forward jets and dijet correlations based on calorimetry have been demonstrated in $p p$ and $p+\mathrm{Pb}$ collisions [38-41]. With the expected detector and luminosity upgrade in future HL-LHC, including for example the charged particle tracking to $|\eta|<4$ and improved granularity of the forward calorimetry in ATLAS and CMS experiments [42,43], a detailed measurement of full jet and jet structure in the forward rapidity should be possible.

The complementarity between midrapidity LHC, forwardrapidity LHC, and midrapidity RHIC can in principle be demonstrated for all commonly-used jet observables. In this paper, we establish this complementary with the dijet $\Delta \phi$ correlation and show its potential to constrain the $\hat{q}$. Assuming integrated luminosity of $10 \mathrm{nb}^{-1}$ for $5.02 \mathrm{TeV}$ $\mathrm{Pb}+\mathrm{Pb}$ collisions expected for LHC-RUN3 [42] and $100 \mathrm{nb}^{-1}$ for $0.2 \mathrm{TeV} \mathrm{Au}+\mathrm{Au}$ collisions expected for sPHENIX at RHIC [34], we estimate the expected statistical precision for dijet $\Delta \phi$ correlations in central collisions. We find that $\Delta \phi$ correlations are dominated by vacuum Sudakov contribution in midrapidity LHC, but are sensitive to medium-induced broadening at forward-rapidity LHC and midrapidity RHIC. We show that the forward-rapidity LHC provides a broader kinematic range for detecting the medium-induced broadening effects.

\section{DIJET CORRELATION IN FORWARD RAPIDITY IN $p p$ AND $A A$ COLLISIONS}

At the leading order, dijets produced in hadronic collisions are back-to-back in the azimuthal plane. However, around $\Delta \phi \approx \pi$ where the pair $p_{\mathrm{T}}$ is much smaller than the individual jet $p_{\mathrm{T}}$, the radiation of soft gluons play an important role, and its contributions need to be resummed to fully describe the experimental data. A Sudakov resummation formalism has been developed in the last few years up to next-to-leading logarithmic order for dijet $\Delta \phi$ correlation [25-28], where the so-called nonglobal logarithmic contributions was found to be important [44-47]. Recently, this formalism has been extended to describe dijet correlations in heavy-ion collisions by including mediuminduced gluon radiation [18-22], which we will briefly describe below.

The dijet cross section in the back-to-back limit in $p p$ collisions can be written as

$$
\begin{aligned}
\frac{d \sigma_{p p}}{d P S}= & \sum_{a b c d} \int \frac{d^{2} b_{\perp}}{(2 \pi)^{2}} e^{-i \vec{q}_{\perp} \cdot \vec{b}_{\perp}} x_{a} f_{a}\left(x_{a}, \mu_{b}\right) x_{b} f_{b}\left(x_{b}, \mu_{b}\right) \\
& \times \frac{1}{\pi} \frac{d \sigma_{a b \rightarrow c d}}{d \hat{t}} \exp [-S(Q, b)],
\end{aligned}
$$

where $d P S=d y_{c} d^{2} p_{T c} d y_{d} d^{2} p_{T d}$ represents the final state phase space, $\mu_{b}=c_{0} / b_{*}$ with $c_{0}=2 e^{-\gamma_{E}}$ and $\gamma_{E}$ the Euler constant. $x_{a}=p_{\mathrm{T}}\left(e^{y_{c}}+e^{y_{d}}\right) / \sqrt{s_{N N}}, x_{b}=p_{\mathrm{T}}\left(e^{-y_{c}}+e^{-y_{d}}\right) /$ $\sqrt{s_{N N}}, Q^{2}=x_{a} x_{b} S$ and $\vec{q}_{\perp}=\vec{p}_{T c}+\vec{p}_{T d} \cdot f_{a}\left(x_{a}, \mu_{b}\right)$ and $f_{b}\left(x_{b}, \mu_{b}\right)$ are the parton distribution functions. The CTEQ14 PDFs [48] are used in the numerical evaluation. $d \sigma_{a b \rightarrow c d} / d \hat{t}$ is the partonic cross section at the leading order. By introducing the $b_{*}$-prescription [49] which sets $b_{*}=b_{\perp} / \sqrt{1+b_{\perp}^{2} / b_{\max }^{2}}$ with $b_{\max }=1.5 \mathrm{GeV}^{-1}$, we separate the Sudakov form factor $S\left(Q, b_{\perp}\right)$ into perturbative and non-perturbative parts in $p p$ collisions:

$$
S\left(Q, b_{\perp}\right)=S_{\text {pert }}\left(Q, b_{\perp}\right)+S_{\mathrm{NP}}\left(Q, b_{\perp}\right)
$$

with the perturbative part defined as,

$$
S_{\text {pert }}\left(Q^{2}, b_{\perp}\right)=\int_{\mu_{b}^{2}}^{Q^{2}} \frac{d \mu^{2}}{\mu^{2}}\left[A \ln \left(\frac{Q^{2}}{\mu^{2}}\right)+B+\left(D_{1}+D_{2}\right) \ln \frac{1}{R^{2}}\right],
$$

where $R$ represents the jet size. We have applied the anti- $k_{t}$ algorithm to define the final state jets in our calculations. Here the coefficients $A, B, D_{1}, D_{2}$ can be expanded perturbatively in terms of powers of $\alpha_{s}$. At one-loop order, $A=C_{A} \frac{\alpha_{s}}{\pi}, B=-2 C_{A} \beta_{0} \frac{\alpha_{s}}{\pi}$ for gluon-gluon initial state, $A=C_{F} \frac{\alpha_{s}}{\pi}, B=\frac{-3 C_{F}}{2} \frac{\alpha_{s}}{\pi}$ for quark-quark initial state, and $A=\frac{\left(C_{F}+C_{A}\right)}{2} \frac{\alpha_{s}}{\pi}, B=\left(\frac{-3 C_{F}}{4}-C_{A} \beta_{0}\right) \frac{\alpha_{s}}{\pi}$ for gluon-quark initial state. $D_{i}$ is $\frac{\alpha_{S}}{2 \pi} C_{F}$ for quark jet, and $\frac{\alpha_{s}}{2 \pi} C_{A}$ for gluon jet. 
In the numerical evaluation of Eq. (3), we have utilized the one-loop running coupling so that these integrals can be carried out analytically. The one-loop running coupling is given by

$$
\alpha_{s}\left(\mu^{2}\right)=\frac{1}{\beta \ln \left(\mu^{2} / \Lambda_{Q C D}^{2}\right)},
$$

where $\beta=\frac{11}{4 \pi}-\frac{n_{f}}{6 \pi}$ with $n_{f}=4$ the active number of quark flavors and $\Lambda_{Q C D}=0.155 \mathrm{GeV}$ in our numerical calculation. Using these expressions, the analytical expressions for the perturbative Sudakov factor can be obtained. For $q+q \rightarrow q+q$ channel, it can be written as:

$$
\begin{aligned}
S_{\mathrm{pert}}^{q+q \rightarrow q+q}\left(Q, b_{\perp}\right)= & \frac{C_{F}}{\pi \beta}\left[-\ln \frac{Q^{2}}{\mu_{b}^{2}}+\ln \frac{Q^{2}}{\Lambda_{Q C D}^{2}} \ln \ln \frac{Q^{2}}{\Lambda_{Q C D}^{2}}\right] \\
& -\frac{C_{F}}{\pi \beta}\left[\ln \frac{Q^{2}}{\Lambda_{Q C D}^{2}} \ln \ln \frac{\mu_{b}^{2}}{\Lambda_{Q C D}^{2}}\right] \\
& -\frac{3 C_{F}}{2 \pi \beta}\left[\ln \ln \frac{Q^{2}}{\Lambda_{Q C D}^{2}}-\ln \ln \frac{\mu_{b}^{2}}{\Lambda_{Q C D}^{2}}\right] \\
& +\frac{C_{F}}{\pi \beta} \ln \frac{1}{R^{2}}\left[\ln \ln \frac{Q^{2}}{\Lambda_{Q C D}^{2}}-\ln \ln \frac{\mu_{b}^{2}}{\Lambda_{Q C D}^{2}}\right] .
\end{aligned}
$$

The perturbative Sudakov factor for other channels can be obtained by changing the color factor accordingly.

For the nonperturbative part of Eq. (2), we follow the prescription given in Ref. [27]

$$
S_{\mathrm{NP}}\left(Q, b_{\perp}\right)=S_{\mathrm{NP}}^{a}\left(Q, b_{\perp}\right)+S_{\mathrm{NP}}^{b}\left(Q, b_{\perp}\right),
$$

where $a$ and $b$ represent the flavors of incoming partons. For a quark, the corresponding nonperturbative factor is given by

$$
S_{\mathrm{NP}}^{q}\left(Q, b_{\perp}\right)=\frac{g_{1}}{2} b_{\perp}^{2}+\frac{g_{2}}{2} \ln \frac{Q}{Q_{0}} \ln \frac{b_{\perp}}{b_{*}},
$$

with $g_{1}=0.212, g_{2}=0.84$ and $Q_{0}^{2}=2.4 \mathrm{GeV}^{2}$. For an incoming gluon, we simply set $S_{\mathrm{NP}}^{g}=\frac{C_{A}}{C_{F}} S_{\mathrm{NP}}^{q}$.

In the $A A$ collisions, we need to add the medium transverse momentum broadening contribution by replacing the vacuum Sudakov factor $S\left(Q, b_{\perp}\right)$ with the medium modified one [18]

$S_{\mathrm{m}}\left(Q, b_{\perp}\right)=S\left(Q, b_{\perp}\right)+\frac{1}{4}\left(\left\langle\Delta q_{\mathrm{T}}^{2}\right\rangle_{c}+\left\langle\Delta q_{\mathrm{T}}^{2}\right\rangle_{d}\right) b_{\perp}^{2}$,

where $\left\langle\Delta q_{\mathrm{T}}^{2}\right\rangle$ is the flavor-dependent averaged transverse momentum broadening square inside the transverse plane, and $\left\langle\Delta q_{\mathrm{T}}^{2}\right\rangle_{g}=\frac{C_{A}}{C_{F}}\left\langle\Delta q_{\mathrm{T}}^{2}\right\rangle_{q}$ between gluon jet and quark jet. Therefore, the broadening for quark jet $\left\langle\Delta q_{\mathrm{T}}^{2}\right\rangle_{q}$ is the only
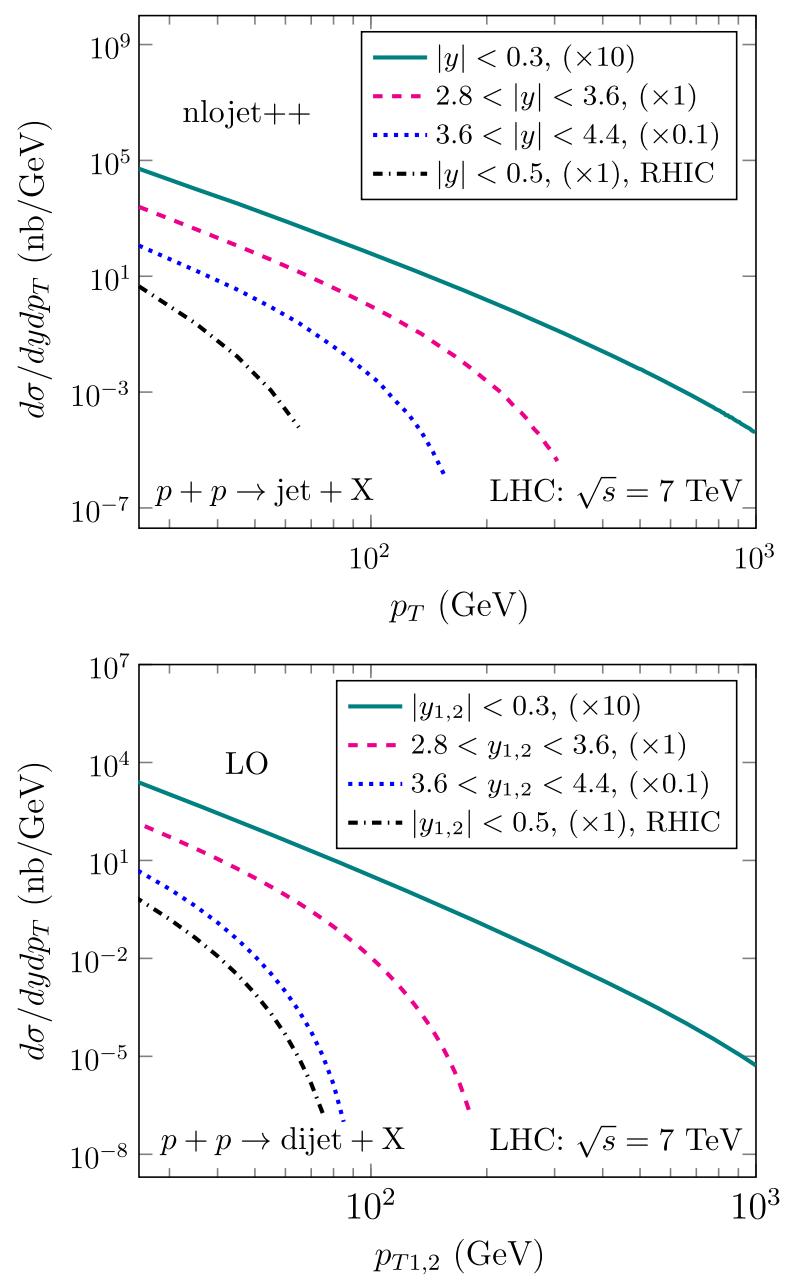

FIG. 1. Inclusive jet cross section at NLO (top panel) and dijet pair cross section at LO with both jets required in the same rapidity range (bottom panel).

free parameter in our numerical study, which is directly proportional to the $\hat{q}$. Following our previous estimation [20], we choose $\left\langle\Delta q_{\mathrm{T}}^{2}\right\rangle_{q}=5 \mathrm{GeV}^{2}$ and $10 \mathrm{GeV}^{2}$ for midrapidity in central $\mathrm{Au}+\mathrm{Au}$ collisions at RHIC and central $\mathrm{Pb}+\mathrm{Pb}$ collisions at the LHC, respectively. The corresponding values of $\hat{q}$ roughly match those extracted in previous studies [17,20], which have sizable uncertainties. For the forward-rapidity LHC, we assume $5<\left\langle\Delta q_{\mathrm{T}}^{2}\right\rangle_{q}<10 \mathrm{GeV}^{2}$, and simply calculate for both 5 and $10 \mathrm{GeV}^{2}$ as boundary conditions.

Figure 1 shows the inclusive jet cross section in $p p$ collisions at NLO [50,51] as well as the dijet pair cross section at LO. We have checked that our NLO calculation reproduces the LHC $p p$ data. The $p_{\mathrm{T}}$ reach for inclusive jet at the LHC, even for the forward rapidity, is much larger than that at RHIC. When the away-side jet is also restricted to be in the same rapidity range, the $p_{\mathrm{T}}$ reach is significantly reduced but nevertheless is still larger than that at RHIC. In principle, one could also consider cases where 

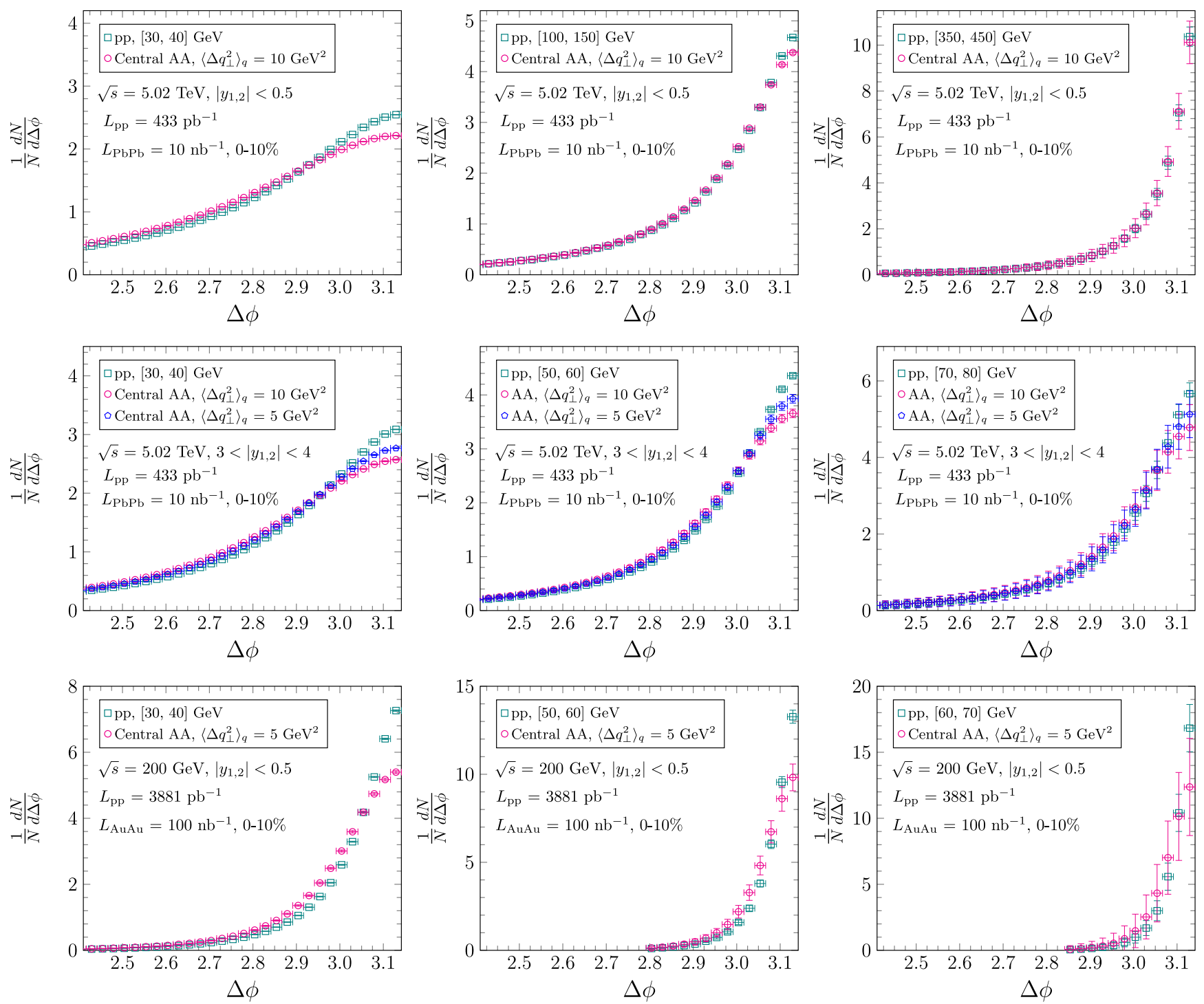

FIG. 2. Prediction for dijet $\Delta \phi$ distributions for the expected luminosity in the midrapidity at the LHC (top row), forward rapidity at the LHC (middle row) and the midrapidity at RHIC (bottom row). The error bars indicate the expected statistical uncertainties.

only one jet is in the forward rapidity, and the other jet is in the central or the backward rapidity. Such forward-central and forward-backward dijet $\Delta \phi$ correlation have greater pair- $p_{\mathrm{T}}$ reach than forward-forward case considered here, but one need to assign two jets different $\left\langle\Delta q_{\mathrm{T}}^{2}\right\rangle$ values matching their local $d N / d \eta$ values. For our first exploratory study, we choose to focus on dijet correlation with both jets restricted in the rapidity range with $3<|y|<4$, thus we can assign the same $\left\langle\Delta q_{\mathrm{T}}^{2}\right\rangle$ to both jets. These forward dijets probe a QGP medium with a temperature comparable to that at RHIC but at larger $Q^{2}$ values.

In Refs. [52-54], the correlation between a jet in the central rapidity region and a forward rapidity jet has been studied. In this work, we focus on the productions of two leading jets in the same rapidity range. As a result, the partonic center-of-mass energy is smaller than those in other configurations. Therefore the vacuum Sudakov factor is minimized. The angular decorrelation in $\mathrm{A}+\mathrm{A}$ collisions is expected to be more sensitive to the medium broadening effect.

For a quantitative estimation of the sensitivity, we assume an integrated luminosity of $10 \mathrm{nb}^{-1}$ for $5.02 \mathrm{TeV} \mathrm{Pb}+\mathrm{Pb}$ expected for LHC-RUN3 [42] and $100 \mathrm{nb}^{-1}$ for $0.2 \mathrm{TeV}$ $\mathrm{Au}+\mathrm{Au}$ expected for the future sPHENIX experiment at RHIC [34]. The $p p$-equivalent luminosity for the $0-10 \%$ most central A + A is estimated as $L_{\mathrm{pp}}^{\mathrm{AA}}=f \times A^{2} L_{\mathrm{AA}} \mathrm{nb}^{-1}$, where $f=R_{\mathrm{AA}}^{\text {cent }} N_{\text {coll }}^{\text {cent }} \sigma_{\mathrm{AA}}^{\text {cent }} /\left(N_{\text {coll }}^{\mathrm{MB}} \sigma_{\mathrm{AA}}^{\mathrm{MB}}\right) \approx 0.2$ accounting for fractional partonic cross section relative to minimum bias events $(\mathrm{MB})$ and suppression of leading jet $R_{\mathrm{AA}}^{\mathrm{cent}} \approx 0.5$. This gives $L_{\mathrm{pp}}^{\mathrm{PbPb}}=87 \mathrm{pb}^{-1}$ and $L_{\mathrm{pp}}^{\mathrm{AuAu}}=776 \mathrm{pb}^{-1}$, respectively. The luminosity for $p p$ reference data is assumed to match that for the $\mathrm{MB} \mathrm{A}+\mathrm{A}$ via 
$L_{\mathrm{pp}}=A^{2} L_{\mathrm{AA}}$. From these luminosity numbers, we could estimate the number of dijet pairs in any $\Delta \phi$ range and the associated statistical uncertainty. The number of dijet pairs is calculated as

$$
N=L_{\text {pp-equivalent }} \int_{\phi_{\min }}^{\phi_{\max }} d \Delta \phi \frac{d \sigma}{d \Delta \phi}
$$

and the associated relative statistical uncertainty is calculated as $\sqrt{1 / N}$.

For our first exploratory study, we have not considered systematic uncertainties associated with UE background subtractions, detector resolution and harmonic flow. However, by choosing jets with small radius (e.g., $R=0.2$ ) and with the UE level expected in central collisions at RHIC [34], these systematic uncertainties were shown to be reduced to a reasonable level for dijets down to $30 \mathrm{GeV}$.

\section{RESULTS}

The top row of Fig. 2 shows the expected dijet $\Delta \phi$ distributions in the midrapidity LHC for several $p_{\mathrm{T}}$ ranges. The corresponding dijet cross section is large (see Fig. 1), but the medium-induced broadening, reflected by the difference between $\mathrm{Pb}+\mathrm{Pb}$ and $p p$, is only visible at $p_{\mathrm{T}}<70 \mathrm{GeV}$, where the full jet reconstruction is challenging due to the large UE fluctuations. At $p_{\mathrm{T}}>100 \mathrm{GeV}$ where the full jet reconstruction is possible, the vacuum Sudakov factor dominates over the medium-induced broadening. This conclusion also agrees with Ref. [18].

The middle row of Fig. 2 shows the forward dijet $\Delta \phi$ distributions in $p p$ and central $\mathrm{Pb}+\mathrm{Pb}$ for the expected luminosity at LHC-RUN3. The medium-induced broadening is more pronounced than for midrapidity dijet at the same $p_{\mathrm{T}}$. As the UE in $3<|y|<4$ is about factor of 1.5-2 smaller than midrapidity [37], the forward dijets could be reconstructed at lower $p_{\mathrm{T}}$ of $30-40 \mathrm{GeV}$. At higher $p_{\mathrm{T}}$, the vacuum Sudakov factor is larger and difference between $p p$ and $\mathrm{Pb}+\mathrm{Pb}$ is reduced. Nevertheless, the statistical precision is good enough for a possible observation up to $p_{\mathrm{T}} \sim 80 \mathrm{GeV}$, especially for the $\left\langle\Delta q_{\mathrm{T}}^{2}\right\rangle_{q}=$ $10 \mathrm{GeV}^{2}$ case.

We observe that the $\Delta \phi$ distributions in the midrapidity are flatter than those in the forward rapidity at the same $p_{\mathrm{T}}$. This is mainly due to two reasons: (1) the Sudakov factor for the $g g$ channel that dominates in the midrapidity, is much larger than that for the $q g$ channel that dominates in the forward rapidity, (2) it is a common practice to set the factorization scale $\mu_{f}=\mu_{b}$ in the Sudakov resummation framework to simplify the formula, which, we find numerically, makes parton shower stronger in the middle rapidity than in the forward rapidity for $g g \rightarrow g g$ channel at low $p_{T}$.

As proposed in Ref. [18], dijet correlation at RHIC, despite its lower $p_{\mathrm{T}}$ reach, are very sensitive to the medium- induced broadening effects due to smaller vacuum radiations. Our results for $0-10 \%$ central $\mathrm{Au}+\mathrm{Au}$ collisions for the expected luminosity are shown in the bottom row of Fig. 2. The much smaller dijet cross section at RHIC compared to forward LHC is largely compensated by the $\times 10$ larger A + A luminosity, and the medium-induced broadening is visible up to $50<p_{\mathrm{T}}<60 \mathrm{GeV}$ range within the expected statistical uncertainties.

To quantify the broadening effect, we calculate the root-mean-square (RMS) width of $\Delta \phi$ distribution:

$$
\Delta \phi_{\mathrm{RMS}}=\sqrt{\frac{\int d \Delta \phi(\Delta \phi-\pi)^{2} \frac{d \sigma}{d \Delta \phi}}{\int d \Delta \phi \frac{d \sigma}{d \Delta \phi}}},
$$

where the range of $\Delta \phi$ integral is chosen from 2.5 to $\pi$ at the LHC. From this, the difference of RMS width between $\mathrm{A}+\mathrm{A}$ and $p p$ collisions is obtained to isolate the medium-induced broadening effects. Figure 3 shows our results of $\Delta D \equiv\left(\Delta \phi_{\mathrm{RMS}}^{\mathrm{AA}}\right)^{2}-\left(\Delta \phi_{\mathrm{RMS}}^{p p}\right)^{2}$ as a function of $p_{\mathrm{T}}$. In general, the $\Delta D$ is largest at low $p_{\mathrm{T}}$ and decreases toward larger $p_{\mathrm{T}}$. The midrapidity LHC results has best statistical precision, but is expected to challenging due to large UE fluctuations. The $\Delta D$ values for forward-rapidity LHC have very good statistical significance. In fact, even for $\left\langle\Delta q_{\mathrm{T}}^{2}\right\rangle_{q}=5 \mathrm{GeV}^{2}$, the statistical significance for forward-rapidity LHC is comparable or even slightly exceeds that for the midrapidity RHIC.

Figures 3 shows that dijets production at RHIC is kinematically limited to about $p_{\mathrm{T}}<60 \mathrm{GeV}$ (see also Fig. 1), although higher- $p_{\mathrm{T}}$ jets, if they were available, would in principle still be sensitive to the medium-induced broadening. In contrast, the dijet production at forward LHC covers a larger $p_{\mathrm{T}}$ reach in a QGP medium that spans a range

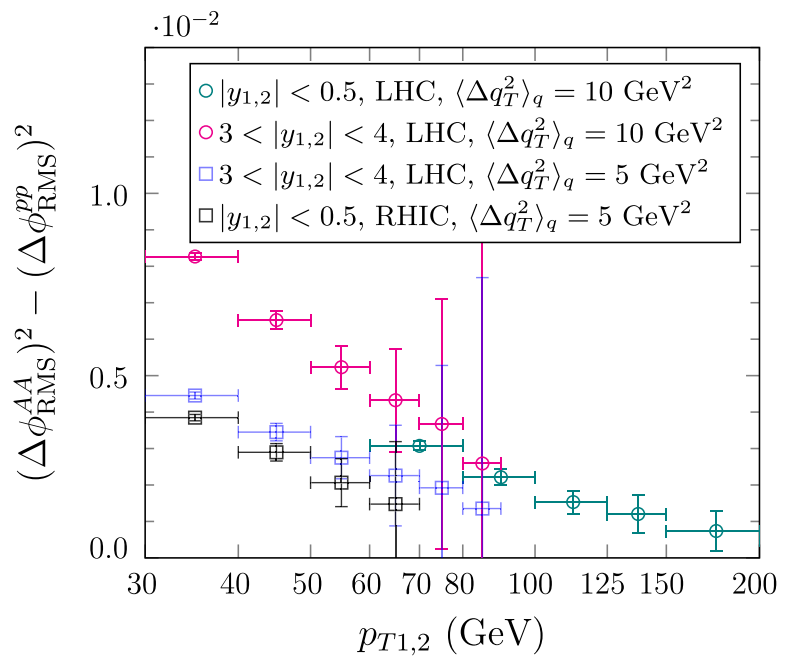

FIG. 3. Difference of RMS width between $\mathrm{A}+\mathrm{A}$ and $p p$ collisions as a function of $p_{\mathrm{T}}$. The error bars indicate the expected statistical uncertainties. 


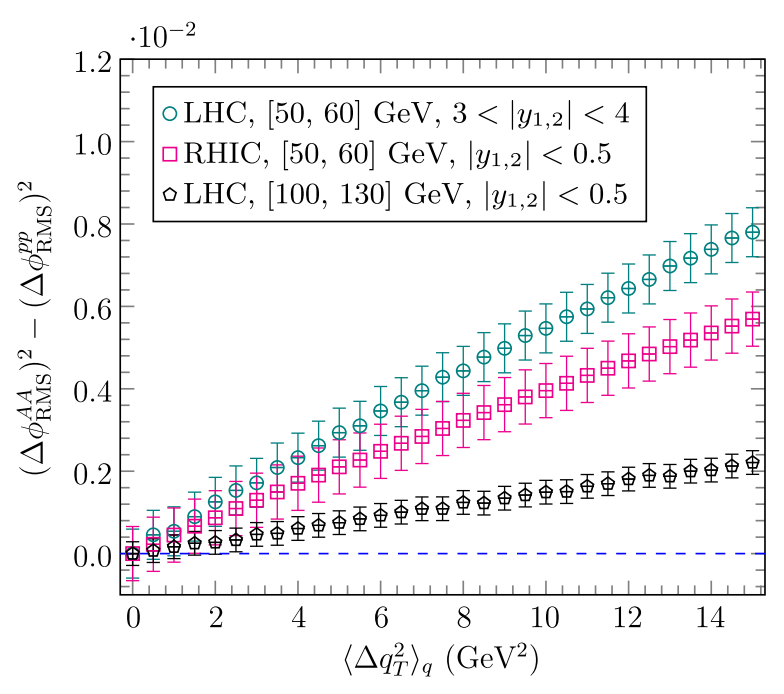

FIG. 4. Difference of RMS width between $\mathrm{A}+\mathrm{A}$ and $p p$ collisions as a function of $\left\langle\Delta q_{\mathrm{T}}^{2}\right\rangle_{q}$. The error bars indicate the expected statistical uncertainties.

of temperature (depending on the rapidity) that could be comparable to that at RHIC. Therefore simultaneous description of dijet correlation (other jet quenching observables as well) in midrapidity RHIC together with the rapidity dependence at the LHC could provide powerful constraints on the jet-medium interactions.

Given the present large uncertainty on the value of $\left\langle\Delta q_{\mathrm{T}}^{2}\right\rangle$, we choose one representative $p_{\mathrm{T}}$ range each for midrapidity RHIC, forward-rapidity LHC and midrapidity LHC, and calculate the corresponding $\Delta D$ as a function of $\left\langle\Delta q_{\mathrm{T}}^{2}\right\rangle$. The results are shown in Fig. 4. The values of $\Delta D$ are comparable between RHIC and forward-rapidity LHC, and both are much larger than that for the midrapidity LHC.
In principle, one could directly extract the value of $\left\langle\Delta q_{\mathrm{T}}^{2}\right\rangle$ once $\Delta D$ is measured.

\section{SUMMARY}

We studied the potential of forward dijet azimuthal correlation at the LHC in the search for the medium-induced $p_{\mathrm{T}}$ broadening effects in heavy ion collisions. We show that the forward dijets at the LHC, enabled by future detector upgrades, are expected to have much better sensitivity compared to midrapidity LHC, due to the steeper jet spectra, smaller vacuum radiations and lower underlying event fluctuations. The expected sensitivity from the upcoming LHC $\mathrm{Pb}+\mathrm{Pb}$ runs is comparable to that of the future $\mathrm{Au}+\mathrm{Au}$ runs at RHIC, but covering a broader $p_{\mathrm{T}}$ range for the dijets and a medium with different temperatures. A direct comparison of the same observable between RHIC and the LHC should provide strong constraints on the collision energy (eventually, the medium temperature) dependence of $\hat{q}$.

\section{ACKNOWLEDGMENTS}

We thank P. Jacobs for useful comments. J. J. is supported by the National Science Foundation Grants No. PHY-1613294 and No. PHY-1913138. S. Y. W. is supported by the Agence Nationale de la Recherche under the Project No. ANR-16-CE31-0019-02. The material of this paper is based upon work partially supported by Lawrence Berkeley National Laboratory, the U.S. Department of Energy, Office of Science, Office of Nuclear Physics, under contract no. DE-AC0205CH11231, and by the Natural Science Foundation of China (NSFC) under Grant No. 11575070.
[1] W. Busza, K. Rajagopal, and W. van der Schee, Annu. Rev. Nucl. Part. Sci. 68, 339 (2018).

[2] M. Gyulassy and M. Plumer, Phys. Lett. B 243, 432 (1990).

[3] X. N. Wang and M. Gyulassy, Phys. Rev. Lett. 68, 1480 (1992).

[4] Y. Mehtar-Tani, J. G. Milhano, and K. Tywoniuk, Int. J. Mod. Phys. A 28, 1340013 (2013).

[5] G. Y. Qin and X. N. Wang, Int. J. Mod. Phys. E 24, 1530014 (2015).

[6] M. Connors, C. Nattrass, R. Reed, and S. Salur, Rev. Mod. Phys. 90, 025005 (2018).

[7] R. Baier, Y. L. Dokshitzer, A. H. Mueller, S. Peigne, and D. Schiff, Nucl. Phys. B483, 291 (1997).

[8] R. Baier, Y. L. Dokshitzer, A. H. Mueller, S. Peigne, and D. Schiff, Nucl. Phys. B484, 265 (1997).

[9] R. Baier, Y. L. Dokshitzer, A. H. Mueller, and D. Schiff, Nucl. Phys. B531, 403 (1998).
[10] B. G. Zakharov, JETP Lett. 63, 952 (1996).

[11] M. Gyulassy, P. Levai, and I. Vitev, Nucl. Phys. B571, 197 (2000).

[12] U. A. Wiedemann, Nucl. Phys. B588, 303 (2000).

[13] X. N. Wang and X.f. Guo, Nucl. Phys. A696, 788 (2001).

[14] P. B. Arnold, G. D. Moore, and L. G. Yaffe, J. High Energy Phys. 06 (2002) 030.

[15] J. Casalderrey-Solana, J. G. Milhano, and U. A. Wiedemann, J. Phys. G 38, 035006 (2011).

[16] J. Casalderrey-Solana, D. C. Gulhan, J. G. Milhano, D. Pablos, and K. Rajagopal, J. High Energy Phys. 10 (2014) 019; 09 (2015) 175(E).

[17] K. M. Burke et al. (JET Collaboration), Phys. Rev. C 90, 014909 (2014).

[18] A. H. Mueller, B. Wu, B. W. Xiao, and F. Yuan, Phys. Lett. B 763, 208 (2016). 
[19] A. H. Mueller, B. Wu, B. W. Xiao, and F. Yuan, Phys. Rev. D 95, 034007 (2017).

[20] L. Chen, G. Y. Qin, S. Y. Wei, B. W. Xiao, and H. Z. Zhang, Phys. Lett. B 773, 672 (2017).

[21] L. Chen, G. Y. Qin, S. Y. Wei, B. W. Xiao, and H. Z. Zhang, Phys. Lett. B 782, 773 (2018).

[22] L. Chen, G. Y. Qin, L. Wang, S. Y. Wei, B. W. Xiao, H. Z. Zhang, and Y. Q. Zhang, Nucl. Phys. B933, 306 (2018).

[23] M. J. Tannenbaum, Phys. Lett. B 771, 553 (2017).

[24] M. Gyulassy, P. Levai, J. Liao, S. Shi, F. Yuan, and X. N. Wang, Nucl. Phys. A982, 627 (2019).

[25] A. Banfi, M. Dasgupta, and Y. Delenda, Phys. Lett. B 665 , 86 (2008).

[26] A. H. Mueller, B. W. Xiao, and F. Yuan, Phys. Rev. D 88, 114010 (2013).

[27] P. Sun, C.-P. Yuan, and F. Yuan, Phys. Rev. Lett. 113, 232001 (2014).

[28] P. Sun, C.-P. Yuan, and F. Yuan, Phys. Rev. D 92, 094007 (2015).

[29] G. Aad et al. (ATLAS Collaboration), Phys. Rev. Lett. 105, 252303 (2010).

[30] S. Chatrchyan et al. (CMS Collaboration), Phys. Rev. C 84, 024906 (2011).

[31] J. Adam et al. (ALICE Collaboration), J. High Energy Phys. 09 (2015) 170.

[32] L. Adamczyk et al. (STAR Collaboration), Phys. Rev. C 96, 024905 (2017).

[33] A. M. Sirunyan et al. (CMS Collaboration), Phys. Lett. B 785, 14 (2018).

[34] A. Adare et al. (PHENIX Collaboration), arXiv:1501.06197.

[35] Y. Akiba et al., arXiv:1502.02730.

[36] J. E. Bernhard, J. S. Moreland, S. A. Bass, J. Liu, and U. Heinz, Phys. Rev. C 94, 024907 (2016).
[37] S. Chatrchyan et al. (CMS Collaboration), Phys. Rev. Lett. 109, 152303 (2012).

[38] G. Aad et al. (ATLAS Collaboration), Phys. Lett. B 748, 392 (2015).

[39] M. Aaboud et al. (ATLAS Collaboration), Phys. Rev. C 100, 034903 (2019).

[40] V. Khachatryan et al. (CMS Collaboration), Eur. Phys. J. C 76, 372 (2016).

[41] A. M. Sirunyan et al. (CMS Collaboration), J. High Energy Phys. 05 (2019) 043.

[42] Z. Citron et al., CERN Yellow Rep. Monogr. 7, 1159 (2019).

[43] J. F. Grosse-Oetringhaus, Nucl. Phys. A982, 170 (2019).

[44] M. Dasgupta and G. P. Salam, Phys. Lett. B 512, 323 (2001).

[45] M. Dasgupta and G. P. Salam, J. High Energy Phys. 03 (2002) 017.

[46] A. Banfi and M. Dasgupta, J. High Energy Phys. 01 (2004) 027.

[47] Y. T. Chien, D. Y. Shao, and B. Wu, J. High Energy Phys. 11 (2019) 025.

[48] S. Dulat, T.-J. Hou, J. Gao, M. Guzzi, J. Huston, P. Nadolsky, J. Pumplin, C. Schmidt, D. Stump, and C.-P. Yuan, Phys. Rev. D 93, 033006 (2016).

[49] J. C. Collins, D. E. Soper, and G. F. Sterman, Nucl. Phys. B250, 199 (1985).

[50] Z. Nagy, Phys. Rev. Lett. 88, 122003 (2002).

[51] Z. Nagy, Phys. Rev. D 68, 094002 (2003).

[52] F. Hautmann and H. Jung, J. High Energy Phys. 10 (2008) 113.

[53] M. Deak, F. Hautmann, H. Jung, and K. Kutak, arXiv:1012. 6037.

[54] M. Deak, F. Hautmann, H. Jung, and K. Kutak, Eur. Phys. J. C 72, 1982 (2012). 